\title{
O envelhecimento sob a perspectiva da vulnerabilidade em saúde: revisão integrativa de literatura
}

\author{
Aging and health vulnerability: \\ integrative literature review
}

FisiSenectus . Unochapecó Ano 3, n. 2 - Jul/Dez. 2015 p. $35-46$

Cezar Grontowski Ribeiro. cezar.ribeiro@ifpr.edu.br

Educador Físico. Mestre em Ciências da Saúde pela Universidade Comunitária da Região de Chapecó-SC. Coordenador do curso de Educação Física do Instituto Federal do Paraná - Campus Palmas. Coordenador do Projeto Melhoria da Saúde e Qualidade de Vida no IFPR Campus Palmas.

Clenise Liliane Schmidt. cleniseschmidt@hotmail.com Enfermeira. Mestre em Ciências da Saúde pela Universidade Comunitária da Região de ChapecóSC. Docente do curso de Enfermagem da Universidade Comunitária da Região de Chapecó-SC.

Aline Mati Shimosaka. alineshimosaka@hotmail.com Acadêmica do curso de Educação Física do Instituto Federal do Paraná - Campus Palmas. Bolsista de Iniciação Cientifica do Projeto Melhoria da Saúde e Qualidade de Vida no IFPR Campus Palmas (PIBIC-FA).

David da Costa. david16-@live.com Acadêmico do curso de Educação Física do Instituto Federal do Paraná - Campus Palmas. Bolsista de Iniciação Cientifica do Projeto Melhoria da Saúde e Qualidade de Vida no IFPR Campus Palmas (PIBIC-FA).

\section{Resumo}

Introdução: As diferenças no modo como cada ser envelhece são influenciadas por vários fatores, como o estado de saúde, gênero, estilo de vida e local de residência, os quais são alvos de estudo em diversas pesquisas atuais. Objetivos: verificar como a literatura científica tem abordado a relação entre envelhecimento e vulnerabilidade em saúde. Metodologia: a revisão integrativa foi realizada a partir da busca integrada dos descritores vulnerabilidade em saúde e envelhecimento, nas bases Scielo e no Portal de Periódicos Capes, entre os registros de 2003 a 2013, realizada em abril de 2013. Resultados: encontrou-se 23 artigos, em que as principais temáticas foram: atenção à saúde; aspectos clínicos do envelhecimento; violência; polimedicação; e institucionalização do idoso. A vulnerabilidade em saúde foi evidenciada durante o processo de envelhecimento, envolvendo: baixas condições socioeconômicas, baixa escolaridade, idosos sem apoio familiar ou institucionalizados, capacidade funcional e cognitiva prejudicadas, presença de patologias crônicas não transmissíveis, uso de polimedicação, falta de políticas públicas para essa população e precariedade do acesso aos serviços de saúde. Considerações finais: os achados permitem identificar fatores que agravam a vulnerabilidade do idoso e consequentemente sinalizam para possíveis estratégias que podem promover a qualidade de vida entre os indivíduos na terceira idade.

\section{Palavras-chave}

Vulnerabilidade em saúde. Envelhecimento. Idoso

\section{Fisiłenectus}




\begin{abstract}
Introduction: the differences in how each be ages are influenced by many fac-tors such as health status, gender, lifestyle and place of residence, which are aims of study in several current searches. Objective: to analyze how the scientific literature has approached the relation between aging and health vulnerability. Methodology: The integrative review was realized with an integrate research about the describers of health vulnerability and aging in the databases of Scielo and in Portal of Periodicals Capes into registers from 2003 to 2013, realized in April 2013. Results: it was found 23 scientific papers whose mainly themes were: health care, clinical aspects of aging, violence, polymedication and institutionali-zation of the elderly. The health vulnerability was evidenced during the process of aging including: low socioeconomic conditions, low schooling, elders without fa-miliar support or homed in institutions, elders with damaged functional or cognitive capacities, the presence of non-transmissible chronic pathologies, the usage of polymedication, lack of public policies for this population and precarious access to health services. Final considerations: the findings allow the researches to identify factors that aggravate the vulnerability of the elders and, consequently, factors that indicate possible strategies to promote a better quality of life for these individuals.
\end{abstract}

\title{
Keywords
}

Health Vulnerability. Aging. Aged.

\section{Introdução}

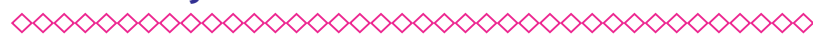

Envelhecer é um processo natural, próprio do ser humano, que possui particularidades e condições específicas para transcorrer com qualidade desejável para manutenção de uma vida amparada em aspectos positivos, nos âmbitos biológico, social, mental, funcional e cognitivo ${ }^{1}$.

0 envelhecimento humano apresenta-se cada vez mais como temática de estudo, devido ao desenho que se traça em âmbito mundial em que ocorre gradual aumento da população idosa, somado à diminuição das taxas de natalidade observadas nas últimas décadas². A expansão da população idosa torna neces-sária uma maior compreensão sobre esse público, quais suas características, interesses, necessidades e, principalmente, vulnerabilidades.

A preocupação com a velhice tem transformado os eixos das políticas públicas e a noção do dever relativo ao Estado para com essa clientela cada vez maior. Pesquisadores destacam que as diferenças no modo como cada ser envelhece são influenciadas por vários fatores como o estado de saúde, gênero, estilo de vida, local de residência, entre outros, e que estes devem ser fonte de pesquisa e análise quando se pensa em indicadores para o envelhecimento humano com qualidade ${ }^{3-5}$.
Procurando postergar ou diminuir o avanço das perdas relativas ao envelhecimento pelo maior período de tempo possível, diversas temáticas têm sido abordadas na literatura ${ }^{6,7}$, tendo destaque a vulnerabilidade devido à necessidade de entendimento das condições multidimensionais que afetam diretamente à saúde dos idosos.

A vulnerabilidade em saúde tem sido compreendida como exposição a riscos que colocam o sujeito em uma determinada condição ou situação que the pode ser prejudicial em uma ou mais variáveis da vida ${ }^{8}$. Como estas não ocorrem somente na esfera individual, mas também em um contexto amplo de sociedade, fomentar o debate acerca da realidade apresentada torna-se primordial.

A vulnerabilidade apresenta-se em três contextos diferentes: individual (decorrente do comportamento pessoal e da qualidade de acesso à informação sobre os problemas de saúde), social (associada ao meio em que se vive, amparada na disponibilidade de recursos materiais, cognitivos e na possibilidade de influência nas decisões políticas e institucionais) e programática (avaliação dos programas de controle das enfermidades, da quantidade de recursos, de gerenciamento e monitoramento das ações no âmbito da atenção à saúde $)^{9}$.

Diante do exposto emergiu a seguinte questão: como a literatura científica tem abordado o 
envelhecimento na perspectiva da vulnerabilidade em saúde?

0 estudo apresenta-se como uma forma de reunir reflexões, relatos, posicionamentos e proposições de variados autores na última década (2003-2013) no que se refere à vulnerabilidade do idoso, resgatando como o ambiente e a sociedade em seu entorno influenciam nessa questão. Assim, o objetivo geral foi verificar como a literatura científica tem abordado o envelhecimento na perspectiva da vulnerabilidade em saúde.

\section{Metodologia}

$\infty \times \infty \times \infty \times \infty \times \infty \times \infty \times \infty \times \infty \times \infty \times \infty \times \infty \times \infty \times \infty \times \infty$

0 presente estudo trata-se de uma pesquisa de base documental, fundamentada nos pressupostos da revisão integrativa de literatura ${ }^{10}$. O levantamento bibliográfico foi realizado, conforme protocolo estruturado e validado, por meio de consulta nas bases de dados da Biblioteca Scielo Brasil e do Portal de Periódicos Capes. A busca ocorreu no período de 4 a 29 de abril de 2013, a partir dos seguintes descritores e combinações: "Vulnerabilidade em Saúde and Envelhecimento"; "Health Vulnerability and Aging"; "Vulnerabilidad en Salud and Envejecimiento".

Utilizou-se como critérios de inclusão: estudos sobre a temática publicados no período dos últimos dez anos (2003 a 2013), nos idiomas inglês, português ou espanhol, oriundos de pesquisas, revisões sistemáticas, reflexões e relatos de experiência, disponíveis na íntegra no formato online e revisadas por pares. Como critérios de exclusão utilizaram-se: estudos duplicados, teses, dissertações, monografia e editoriais, estudos indisponíveis no formato online completo.

Para organização da pesquisa estabeleceramse as seguintes etapas: a) busca do quantitativo de trabalhos apresentados na(s) base(s) a partir dos critérios de inclusão e exclusão determinados; b) leitura individual dos títulos e resumos de todos os trabalhos selecionados; c) busca do formato completo dos artigos, os quais foram salvos em pasta específica; d) leitura flutuante dos artigos completos, de modo independente (duplo cego), em que os trabalhos excluídos por ambos foram retirados da análise.
Para análise final foi realizada a leitura minuciosa dos trabalhos selecionados em sua íntegra, com o objetivo de constatar a aderência ao objetivo deste estudo. Os achados foram organizados em uma tabela no Microsoft Word ${ }^{\circledR}$ e analisados a partir dos itens relacionados na matriz, em que foram dispostas as seguintes informações de cada artigo: ano de publicação, título do artigo, autores, periódico e país em que foi publicado, base em que foi localizado, categoria de pesquisa, natureza/abordagem da pesquisa, objetivos do estudo, referencial teórico utilizado, metodologia, resultados, observações pertinentes. Dentro de cada item as ideias foram agrupadas por similaridade, de modo a produzir uma síntese de forma narrativa.

Inicialmente, foram encontrados 60 artigos potencialmente relevantes (Scielo Brasil $=2$; Portal de Periódicos Capes $=58$ ). Foram excluídos 19 estudos com base nos critérios, tendo em vista que sete não estavam apresentados em formato de artigo científico, cinco não haviam sido revisados por pares, seis não atendiam aos critérios de idioma (português, inglês, espanhol) e um não atendia ao período estabelecido para consulta (2003-2013).

Na sequência realizou-se a leitura na íntegra dos 41 artigos selecionados na primeira etapa. Observou-se que um artigo estava repetido em ambas as bases de consulta e 17 artigos estavam fora do tema pesquisado, ou seja, não abordavam a temática. Dessa forma, a revisão identificou 23 artigos de interesse. 0 processo de seleção está ilustrado na Figura 1.

\section{Resultados e Discussão}

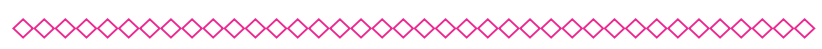

Inicialmente, se analisou o perfil das produções sobre a temática. Em relação à temporalidade, foram identificados trabalhos publicados entre os anos de 2006 a 2012, sendo que a maioria (78,25\%) das publicações concentrou-se no período de 2007 a 2010, com maior incidência neste último, conforme pode ser observado na Tabela 1.

Destas, a maioria dos trabalhos está vinculada ao Portal de Periódicos Capes ( $n=21-91,3 \%$ ), enquanto poucos trabalhos foram encontrados no 
Scielo Brasil ( $n=2-8,7 \%)$. Em relação ao idioma, 20 trabalhos (86,96\%) encontravam-se no idioma português e somente três estudos $(13,04 \%)$ foram apresentados em inglês, todos norte-americanos.

Quanto ao periódico de origem, evidenciou-se que a maioria dos estudos foram apresentados na Revista Ciência \& Saúde Coletiva, contudo, outros periódicos registraram manuscritos sobre a temática. (Tabela 2)

No que se refere à categoria dos estudos, foram encontrados 15 artigos originais (62,5\%) e nove revisões de literatura (37,5\%). No método de abordagem empregado, observou-se que predominam as pesquisas qualitativas ( $\mathrm{N}=12-50 \%)$, seguida pelos trabalhos quantitativos ( $\mathrm{N}=8-33,33 \%)$ e por pesquisas quanti e qualitativas ( $N=4-16,67 \%)$.

A discussão que segue foi pautada nos resultados que apresentaram maior recorrência nos artigos selecionados, as quais foram: atenção à saúde ( $n=14-60,87 \%)$; aspectos clínicos do envelhecimento $\quad(n=12 \quad-52,17 \%)$; violência ( $n=7-30,43 \%)$; polimedicação $(n=3-13,14 \%)$; e institucionalização do idoso ( $n=2-8,70 \%)$, como pode ser verificado na Figura 2.

\section{A vulnerabilidade em saúde}

A vulnerabilidade nos artigos revisados é conceituada como a capacidade do indivíduo sofrer dano em resposta a um estímulo e envolve os planos biológico/individual, social e programático/institucional. Vista dessa forma, qualquer mudança que ocorra em um desses planos repercutirá consequências nos demais, já que a vulnerabilidade rompe com a universalidade dos determinantes de saúde ${ }^{6,7}$. Alguns estudos trazem, ainda, a vulnerabilidade como predisposição ou risco de fragilização ${ }^{11-13}$.

A vulnerabilidade abrange o grau e qualidade da informação que os indivíduos dominam sobre o problema e a capacidade de elaborar respostas às situações e adotá-las em sua vida cotidiana ${ }^{6}$. Assim, pode estar associada a mudanças de determinantes econômicos, sociais, ambiente físico, individuais, comportamen-tais, serviços sociais e de saúde 7 . Aliado a essa caracterização, consideram-se vulneráveis as populações que apresentam maior suscetibilidade para sofrer danos ou mudanças desses determinantes, incluindo excepcionalmente os idosos.

\section{Envelhecimento e vulnerabilidade: influências da atenção à saúde}

A atenção à saúde foi o principal aspecto abordado nos artigos em questão, tratando, principalmente, dos níveis primário e secundário de serviços de saúde. Dentre os 23 artigos analisados, 14 abordam os programas oferecidos nos diferentes níveis de Atenção à Saúde 6,7,13-24.

Destacou-se no contexto da atenção à saúde a importância da Estratégia de Saúde na Família (ESF) como estratégia com potencial positivo para o enfrentamento da vulnerabilidade durante 0 envelhecimento, uma vez que ela propõe vínculos entre usuário e profissional, favorecendo um acompanhamento mais sistemático e englobando as três dimensões do cuidado: promoção, prevenção e recuperação da saúde ${ }^{6,7,13,14,16,19,23}$.

A ESF configura-se como uma estratégia de reorientação do modelo de assistência, realizada pela implantação de equipes multiprofissionais em Unidades Básicas de Saúde e requer, especialmente, a aproximação à realidade dos usuá-rios e famílias ${ }^{25}$.

Por outro lado, para que o atendimento à pessoa idosa englobe os princípios do SUS (integralidade, equidade e universalidade) fazse necessária uma abordagem multidimensional do idoso, por meio da atenção multiprofissional. Essa proposta inclui o Núcleo de Apoio à Saúde da Família (NASF), a Equipe de Saúde Bucal e a ESF a nível de Atenção Básica6,13,14,16,19. Além disso, quando obser-vada a atenção secundária (que inclui hospitais e instituições de longa permanência de idosos), a atenção multidimensional faz-se tão importante quanto na Atenção Básica. $\mathrm{E}$, ao contrário do que seria adequado, ou seja, que a institucionalização proporcionasse amplo atendimento de saúde, os estudos indicam que os idosos têm menos acesso a algumas áreas profissionais quando institucionali-zados, como psicólogos, fisioterapeutas, psiquiatras, entre outros, fazendo com que a internação torne-se 
mais nociva que positiva à saúde e qualidade de vida ${ }^{13,16,20,21,23,26}$.

Para que a população idosa tenha garantia de acesso aos serviços de saúde, às redes sociais de apoio e aos serviços que atendam às necessidades indi-viduais, várias políticas públicas têm sido criadas com a intenção de melhorar a qualidade de vida desse grupo populacional. Entre elas, as de maior destaque são a Política Nacional do Idoso (PNI), de 1994, o Estatuto do Idoso, de 2003, e a Política Nacional de Saúde da Pessoa Idosa, de 2006. Elas abordam enfaticamente os principais agravos que ocorrem na fase do envelhecimento, os direitos e garantias de acesso aos serviços públicos. Além destas, a Política Nacional de Redução de Morbimortalidade por Acidentes e Violências (PNRMAV), de 2001, e o Pacto pela Saúde também fazem referência à pessoa idosa ${ }^{20}$.

Dos 23 artigos estudados nesta revisão, nove deles trazem uma abordagem sobre as políticas públicas específicas para idosos, relatadas no parágrafo acima $6,12,13,15-17,19,20,27$.

Nesse contexto, o envelhecimento da população está associado a mudanças no cenário social, econômico e de saúde, ocasionando maior demanda de serviços de saúde e um impacto econômico nas políticas públicas relacionadas diretamente aos idosos $6,7,14,16,21$. Devido a esses aspectos, a vulnerabilidade tem sido vinculada ao tema envelhecimento, principalmente no sentido de diagnosticar as dificuldades encontradas pelos idosos para viver mais dignamente e com maior participação social, além de encontrar meios que permitam melhorar a qualidade de vida dessa parcela da população, criando estratégias e ações facilitadoras do viver saudável.

\section{Aspectos clínicos do envelhecimento e da vulnerabilidade}

No Brasil, o aumento da expectativa de vida não tem sido acompanhado pela melhoria da qualidade de vida, principalmente ao considerar a população de baixa renda e de maior risco social ${ }^{23}$. Nesse sentido, evidencia-se 0 aumento da população idosa acompanhada do aumento do registro de morbimortalidade por doenças crônicas e implicações cognitivas e funcionais nessa população 7,14,18,21.
Dentre as características clínicas mais prevalentes durante o processo de envelhecimento, destacam-se algumas condições limitantes que favorecem a fragilidade/vulnerabilidade dos idosos. Os aspectos mais citados nesse contexto são problemas odontológicos, declínio físico, maior incidência de quedas e fraturas, incapacidade funcional, sofrimento psíquico, maior risco para suicídio, incidência de disfunções sexuais e maior incidência de casos relatando problemas de incontinência urinária $6,7,14,16,20-24,26-28$.

Além disso, alguns fatores têm sido associados ao envelhecimento da população, interferindo diretamente na avaliação clínica do idoso. Discutem-se nos artigos, aspectos fisiológicos (sensoriais, gastrointestinais, metabólicos, cardiovasculares e renais), psicossociais (mudanças nos hábitos alimentares) e físicos (diminuição da visão, incapacidades físicas e mobilidade) ${ }^{16,18}$.

Essa realidade dá-se por meio da alta prevalência de idosos acometidos por doenças crônicas não transmissíveis, com declínio físico e funcional. Vários autores apontam para uma tendência dessas características, sendo estabelecido um perfil comum para o adoecimento desse grupo populacional12,13,16,21,29,30.

Segundo parte dos estudos, o envelhecimento está mais ligado à questão do gênero feminino, pois a expectativa de vida das mulheres supera a dos homens devido à maior vulnerabilidade dos homens. Essa ideia está ligada a evidências de maior procura dos serviços de saúde pelas mulheres, além de hábitos de vida mais saudáveis adotados por estas $7,13,16,21,27,30$

Ainda há outros aspectos evidentes na população idosa estudada, como maior concentração de indivíduos com baixa escolaridade, baixa renda e grande prevalência de doenças crônicas não transmissíveis, aspectos que predispõem o idoso à vulnerabilidade $7,13,16,21,27,30$.

\section{A violência contra o idoso e a vulnerabilidade}

A violência praticada contra idosos foi amplamente abordada nos artigos científicos desta revisão. Entre as situações de maior risco, as questões familiares/domiciliares são apontadas 
como as condições mais recorrentes para a prática da violência $6,13,17,27,31,32$

Minayo $^{31}$ é uma das autoras que estuda questões relacionadas à violência contra o idoso. Ela aponta para situações de negligência e maus tratos ocorridas no ambiente familiar. A violência é considerada um fenômeno humano traduzido em atos, que visa prejudicar, ferir, mutilar ou matar o outro, podendo ser física, sexual, psicológica ou espiritual.

Outros quatros artigos também trazem o familiar como principal autor da prática de violência contra idosos e o ambiente familiar como o ambiente de maior risco para ocorrência dos casos de violência 13,17,27,32. A literatura destaca, ainda, a necessidade de inserir o tema violência nas políticas públicas, com o objetivo de garantir o direito de integridade física e psicológica dos idosos ${ }^{12,17}$.

Compreende-se que o tema violência vem sendo pesquisado e discutido nos últimos anos, promovendo e subsidiando novas abordagens e a adequação de políticas que tratam do envelhecimento, mas carece de ampliação e proposição de políticas e ações efetivas para se concretizar, de forma eficiente, a redução da violência contra o idoso, diminuindo a vulnerabilidade destes nessa variável.

\section{Polimedicação e vulnerabilidade dos idosos}

Outro aspecto associado ao envelhecimento é a vulnerabilidade para polimedicação. Estudos apontam que o uso simultâneo de medicamentos de diferentes classes farmacológicas é prevalente em idosos ${ }^{11,21,30}$. Estes afirmam que a polimedicação leva muitos desses indivíduos a usar incorretamente os fármacos, devido à grande diversidade de medicamentos utilizada e disponível ao idoso.

Entre os fatores de risco mais prevalentes para polimedicação, dois estudos citam viver sozinho, ser do gênero feminino e possuir baixa escolaridade como aspectos diretamente associados a essa conduta ${ }^{11,30}$. Além disso, a literatura con-sultada destaca que a incidência de doenças crônico-degenerativas é uma das maiores responsáveis pela grande prevalência de idosos que se polimedicam ${ }^{11,21,30}$. Os autores apontam para as doenças do sistema cardiovascular como principais responsáveis pelo uso de medicamentos.

Um dos estudos revisados ainda traz a polimedicação como um tema em expansão, que precisa ser estudado de maneira crescente, já que o uso simultâ-neo de medicamentos está associado a iatrogênias ${ }^{11}$.

Um estudo realizado no Brasil constatou que $56,6 \%$ dos idosos utiliza receita com pelo menos quatro medicamentos de uso contínuo. De acordo com os autores, o idoso está mais vulnerável aos efeitos colaterais dos medicamentos, podendo inclusive, ampliar a severidade das patologias já existentes. 0 aumento dos distúrbios mentais também aumenta a vulnerabilidade do idoso, os quais re-querem o uso frequente de medicamentos psiquiátricos ${ }^{33}$.

De maneira geral, os artigos que abordam a questão da polimedicação afirmam a alta incidência dessa prática em idosos, reforçando a necessidade de realizar estudos e intervenções que disseminem o conhecimento sobre os riscos do uso simultâneo de medicamentos e racionalização da medicação dos idosos.

Institucionalização do idoso e vulnerabilidade

A institucionalização de idosos tem sido associada às modificações na estrutura das famílias e à dinâmica da sociedade, em que há inserção de um maior número de integrantes das famílias no mercado de trabalho, provocando mudanças no contexto da família e a ausência de cuidador familiar ${ }^{20}$.

Dos artigos revisados, dois apontam para o tema da institucionalização dos idosos com maior ênfase e abordam aspectos relacionados ao perfil clínico e social desses idosos, à estrutura das instituições, aos profissionais que compõem a equipe e à sua dinâmica de trabalho. Outro aspecto abordado é a crescente demanda de idosos que são institucionalizados e o aumento do número de instituições de longa permanência no Brasil ${ }^{20,21}$. 
Entretanto, o dado mais evidente sobre a institucionalização está associado à prevalência da incapacidade funcional ou dependência. Quando há presença de uma série de incapacidades físicas ou cognitivas no idoso, estas exigem maior demanda de serviço e comprometimento do grupo familiar ${ }^{20,21}$. Muitas vezes, não há um integrante da família que possa assumir o cuidado desse idoso, 0 que aumenta a procura pela institucionalização. Em outros casos, o idoso reside sozinho, sem família que possa auxiliá-lo, repercutindo, da mesma forma, na procura por institucionalização conforme 0 avanço da idade e das necessidades de saúde ${ }^{21}$.

Apesar de os estudos apresentarem a institucionalização como fator negativo pelo afastamento da dinâmica familiar que ela provoca, estudos também apontam resultados positivos, afirmando que os idosos inseridos nesse meio re-cebem cuidados profissionais adequados, de acordo com suas necessidades de saúde ${ }^{21}$. Por outro lado, há estudos que se opõem a essa afirmação, demonstrando que, frequentemente, as instituições de longa permanência não possuem re-cursos humanos capacitados para atender as reais necessidades dessa população de perfil diferenciado $7,13,14,20$.

$\mathrm{Na}$ análise dos serviços oferecidos pelas instituições de longa permanência de idosos, na maioria das instituições estudadas o cuidado fica restrito à equipe de enfermagem. Em alguns casos há inserção de outras áreas profissionais, como médico e odontólogo ou, ainda, de equipe multiprofissional, possibilitando atendimento multidimensional ao idoso. Com isso se discute a repercussão da institucionalização em modelos, às vezes, inadequados para atender as necessidades dos idosos ali inseridos. Isto leva à necessidade de rever a dimensionalidade dos cuidados oferecidos nesses locais, como forma de otimizar o uso dessas instituições, ofertando um serviço de qualidade, que permita uma vida digna ao indivíduo ${ }^{14,21}$.

Todo o exposto leva a crer que a institucionalização apresenta aspectos positivos e negativos, precisando ser vista a partir de um continuum, em que se pesem as questões que implicam em maior vulnerabilidade entre os idosos, como a possibilidade de um acompanhamento mais próximo das equipes de saúde, um ambiente favorável ao desenvolvimento de hábitos saudáveis, que permitam uma vida mais longa e feliz.

Por fim, compreende-se que as questões geradoras da vulnerabilidade afetam diretamente a percepção do sujeito acerca de sua qualidade de vida. A preocupação com a satisfação com a vida, evitando as situações que causam dor e sofrimento, deve ser meta constante das equipes de saúde, já que pessoas mais vulneráveis acabam tornando-se mais suscetíveis a males relacionados com essa realidade, interferindo negativamente na forma como veem sua vida. Assim, as equipes de saúde devem priorizar situações que permitam não somente diminuir os aspectos relacionados à vulnerabilidade, mas também ações que possam servir como facilitadoras e mantenedoras da qualidade de vida.

\section{Considerações finais}

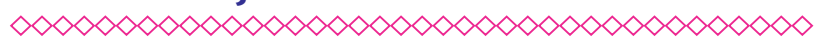

A literatura científica atual aponta que a vulnerabilidade em saúde é evidenciada durante o processo de envelhecimento, especialmente devido às baixas condições socioeconômicas e escolaridade dos idosos, à falta do apoio familiar, à capacidade funcional e cognitiva prejudicadas, bem como pelo acometimento de doenças crônicas não transmissíveis, que somadas às falhas na rede de atenção à saúde repercutem no uso de polimedicação e na necessidade de institucionalização do idoso.

Os estudos permitem identificar aspectos específicos da vulnerabilidade entre os idosos e as interfaces com o ambiente e sociedade em que estão inseridos, bem como aspectos que carecem de maiores investigações e implantação de estratégias de enfrentamento para promoção da saúde do idoso.

Um fator limitante do estudo pode ser relacionado com a pesquisa em ape-nas duas plataformas de busca e pelo fato de serem selecionados somente artigos científicos.

Constatou-se que existem vários aspectos geradores da vulnerabilidade inerente à saúde 
e que são necessárias políticas públicas mais eficazes para suprir as carências populacionais no que se refere, principalmente, às questões elencadas neste estudo. Desse modo, entendemos que somente será possível construir uma sociedade mais justa e igualitária, quando forem aliadas a ética, políticas públicas e pesquisas para conhecimento da realidade eficazes. É preciso compreender que o risco à vulnerabilidade ocorre não somente nas questões materiais, mas, principalmente, na organização social e de relações humanas.

Em síntese, as pesquisa demonstram que é necessário investimento em pesquisas, políticas públicas e tecnologias que minimizem a vulnerabilidade nesse grupo social. Nesse contexto, destacam-se a necessidade e maior preocupação com a violência contra o idoso e sua institucionalização.

Agência de Fomento: Programa Institucional de Bolsas de Iniciação Científica do IFPR Fundação Araucária.

\section{Referências}

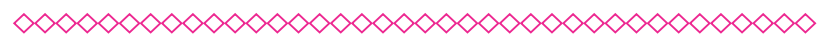

1. Veras RP. Envelhecimento populacional contemporâneo: demandas, desafios e inovações. Rev. Saúde Públ. 2009; 3(3): 548-554.

2. Instituto Brasileiro de Geografia e Estatística. Diretoria de Pesquisas. Síntese de Indicadores Sociais. Rio de Janeiro: IBGE, 2010. Disponível em: <http://www.ibge.gov.br/>.

3. Nelson ME, Rejeski WJ, Blair SN, Duncan PW, Judge JO, King AC, et al. Physi-cal Activity and Public Health in Older Adults: Recommendation From the Ameri-can College of Sports Medicine and the American Heart Association. Med Sci. Sports Exserc. 2007; 39(8): 1435-1445.

4. Nasri F. O envelhecimento populacional no Brasil. Einstein (São Paulo). 2008; 6 (supl1):S4-S6.

5. Leite LEA. Envelhecimento, estresse oxidativo e sarcopenia: uma abordagem sistêmica. Rev. Bras. Geriatr. Gerontol. 2012; 15(2): 365-380.
6. Paz AA, Santos BL, Eidt OR. Vulnerabilidade e envelhecimento no contexto da saúde. Acta Paul. Enferm. 2006; 19(3): 338-342.

7. Braga LS, Macinko J, Proietti FA, César CC, Lima-Costa MF. Diferenciais intraurbanos de vulnerabilidade da população idosa. Cad. Saúde Pública. 2010; 26(12): 2307-2315.

8. Spink MJP. Trópicos do discurso sobre risco: risco-aventura como metáfora na modernidade tardia. Cad. Saúde Pública. 2001;17(6):1277-1311.

9. Ayres JRCM, França Junior I, Calazans G, Salletti $H$. Vulnerabilidade e prevenção em tempos de Aids. In: Barbosa R, Parker R, organizadores. Sexualidade pelo avesso: direitos, identidades e poder. Rio de Janeiro: Relume Dumará; 1999.

10. Ganong LH. Integrative reviews of nursing research. Res Nurs Health.1987 Fev;10(1):1-11.

11. Galato D, Silva ES, Tiburcio LS. Estudo de utilização de medicamentos em idosos residentes em uma cidade do sul de Santa Catarina (Brasil): um olhar so-bre a polimedicação. Ciênc. Saúde Colet. 2010; 15(6): 2899-2905.

12. Souza ER, Minayo MC. Inserção do tema violência contra a pessoa idosa nas políticas públicas de atenção à saúde no Brasil.Ciênc. Saúde Colet. 2010; 15(6): 2659-2668.

13. Apratto Júnior PC. A violência doméstica contra idosos nas áreas de abrangência do Programa Saúde da Família de Niterói (RJ, Brasil). Ciênc. Saúde Colet. 2010; 15(6): 2983- 2995.

14. Feuerwerker LCM, Emerson EM. A contribuição da atenção domiciliar para a configuração de redes substitutivas de saúde: desinstitucionalização e transfor-mação de práticas. Rev. Panam. Salud Publ. 2008; 24(3):180-188.

15. Assis M, Hartz ZMA, Pacheco LC, Valla VV. Avaliação do projeto de promoção da saúde do Núcleo de Atenção ao idoso. Interface (Botucatu). 2009; 13(29):367-382.

16. Nunes DP, Nakatani AYK, Silveira EA, Bachion MM, Souza MR. Capacidade funcional, condições socioeconômicas e de saúde de idosos atendidos por equi-pes de Saúde da Família de Goiânia(GO, Brasil).Ciênc. Saúde Colet. 2010;15(6):2887-2898. 
17. Valadares FC, Souza ER. Violência contra a pessoa idosa: análise de aspec-tos da atenção de saúde mental em cinco capitais brasileiras. Ciênc. Saúde Colet. 2010;15(6):2763-2774.

18. Vilarino MAM, Lopes MJM, Bueno ALM, Brito MRV. Idosos vacinados e nãovacinados contra influenza: morbidade relatada e aspectos sócio demográficos, Porto Alegre-RS-Brasil. Ciênc. Saúde Colet. 2010;15(6):2879-2886.

19. Costa JMBS, Silva MRF, Carvalho EF. Avaliação da implantação da atenção à hipertensão arterial pelas equipes de Saúde da Família do município do Recife (PE, Brasil). Ciênc. Saúde Colet. 2011; 16(2):623-633.

20. Ferreira RC, Wolf C. Atenção odontológica e práticas de higiene bucal em instituições de longa permanência geriátricas. Ciênc. Saúde Colet. 2011; 16(4): 2322-2333.

21. Carvalho MP, Luckow ELT, Siqueira FV. Quedas e fatores associados em idosos institucionalizados no município de Pelotas (RS, Brasil). Ciênc. Saúde Colet. 2011;16(6):2945-2952.

22. Simões ACA, Carvalho DM. A realidade da saúde bucal do idoso no Sudeste brasileiro.Ciênc. Saúde Colet. 2011;16(6):2975-2982.

23. Ursine PGS, Cordeiro HA, Moraes CL. Prevalência de idosos restritos ao domicílio em região metropolitana de Belo Horizonte- Minas Gerais-Brasil. Ciênc. Saúde Colet. 2011; 16(60): 2953-2962.

24. Volkmer C, Montirelli M, Reibnitz KS, Bruggemann OM, Sperandio FF. Incontinência Urinária feminina: revisão sistemática de estudos qualitativos. Ciênc. Saúde Colet. 2012; 17(10): 2703-2715.

25. Fonseca AFQ. Ambiente e saúde: visão de profissionais da saúde da família. Ambient. soc. 2012;15(2):133-150.

26. Rohden F. Diferenças de gênero e medicalização da sexualidade na criação do diagnóstico das disfunções sexuais. Rev. Estud. Fem.. 2009; 17(1): 89-109.
27. Minayo MCS, Souza ER, Paula DR. Revisão sistemática da produção acadêmica brasileira sobre causas externas e violências contra a pessoa idosa. Ciênc. Saúde Colet. 2010; 15(6): 2719-2728.

28. Fortes-Burgos ACG, Neri AL, Cupertino APFB. Eventos estressantes, estratégias de enfrentamento, autoeficácia e sintomas depressivos entre idosos residentes na comunidade.Psicol. refl. crít. 2008; 21(1): 74-82.

29. Nieiro MBP, Alencar GP, Bergamachi DP. Morbi-mortalidade por doenças do aparelho respiratório em idosos antes e após a introdução da vacina contra influenza. São Paulo Perspec. 2008; 22(2): 46-54.

30. Medeiros EFF, Moraes C, Karnikowski M, Nóbrega OT, Karnikowski MGO. Intervenção interdisciplinar enquanto estratégia para o Uso Racional de medica-mentos em idosos. Ciênc. Saúde Colet. 2011; 16(7): 3139-3149.

31. Minayo MCS, Cavalcante FG. Suicídio entre pessoas idosas: revisão da litera-tura. Rev.Saúde Públ. 2010; 44(4): 750-757.

32. Agra do Ó A, Souza RN, Costa T, Almeida VB. $A$ violência contra idosos na mídia: uma reflexão sobre a produção dos sentidos. Revista FAMECOS. 2010; 17(1): 121-129.

33. Gomes HO, Caldas CP. Uso inapropriado de medicamentos pelo idoso: polifarmácia e seus efeitos. Rev Hosp Univ Pedro Ernesto. 2008; 7(1):88-99. 


\section{Anexos}

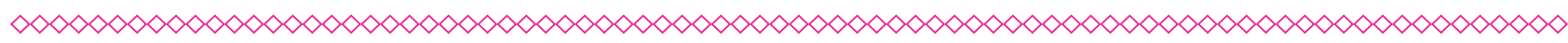

Figura 1 - Procedimentos para construção da revisão integrativa

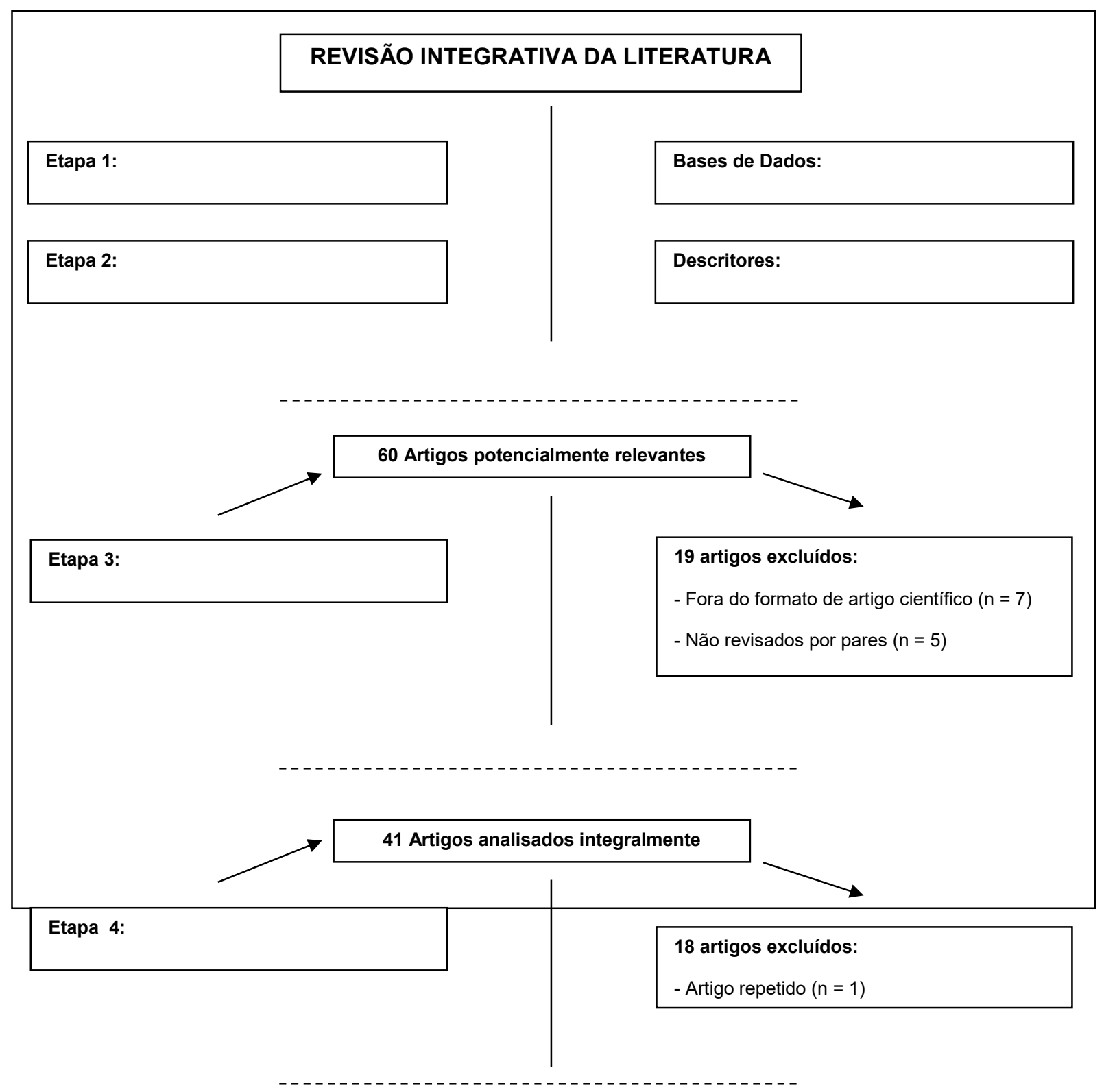

Resultado: 23 Artigos de interesse

(clique para voltar ao texto) 
Tabela 1 - Publicações no período 2006-2012

\begin{tabular}{rrr}
\hline Ano & Qtde & $\%$ \\
\hline 2006 & 2 & 8,70 \\
2007 & 3 & 13,04 \\
2008 & 4 & 17,39 \\
2009 & 3 & 13,04 \\
2010 & 8 & 34,78 \\
2011 & 2 & 8,70 \\
2012 & 1 & 4,35 \\
\hline & 23 & 100,00 \\
\hline
\end{tabular}

(clique para voltar ao texto)

Tabela 2 - Periódicos com publicações na temática no período investigado

\begin{tabular}{lcr}
\hline Periódico & Quantidade & \% \\
\hline Rev. Ciência e Saúde Coletiva & 14 & 60,87 \\
Rev. Ciência e Saúde Pública & 2 & 8,70 \\
Rev. Saúde Pública & 1 & 4,35 \\
Interface, comunicação, saúde e educação & 1 & 4,35 \\
Estudos Feministas & 1 & 4,35 \\
Psicologia: reflexão e crítica & 1 & 4,35 \\
Rev. Panamericana de Salud Publica & 1 & 4,35 \\
São Paulo em Perspectiva & 1 & 4,35 \\
Rev. FAMECOS & 1 & 4,35 \\
\hline & 23 & 100,00 \\
\hline
\end{tabular}


Figura 2 - Resumo da matriz dos artigos científicos encontrados nas plataformas de busca

\begin{tabular}{|c|c|c|c|c|}
\hline & TÍTULO & AUTOR(ES) & ANO & PERIÓDICO \\
\hline 1 & $\begin{array}{l}\text { Estudo de utilização de medicamentos em idosos residentes em uma } \\
\text { cidade do sul de Santa Catarina (Brasil): um olhar sobre a polimedicação. }\end{array}$ & Dayani Galato et al. & 2007 & $\begin{array}{l}\text { Revista Ciência } \\
\text { Saúde Coletiva }\end{array}$ \\
\hline 2 & $\begin{array}{l}\text { Inserção do tema violência contra a pessoa idosa nas políticas públicas } \\
\text { de atenção à saúde no Brasil. }\end{array}$ & $\begin{array}{l}\text { Edinilsa Ramos de } \\
\text { Souza, Maria Cecília } \\
\text { de Souza Minayo. }\end{array}$ & 2010 & $\begin{array}{l}\text { Revista Ciência } \\
\text { Saúde Coletiva }\end{array}$ \\
\hline 3 & $\begin{array}{l}\text { Atenção odontológica e práticas de higiene bucal em instituições de } \\
\text { longa permanência geriátricas. }\end{array}$ & $\begin{array}{l}\text { Raquel Conceição } \\
\text { Ferreira et al }\end{array}$ & 2006 & $\begin{array}{l}\text { Revista Ciência } \\
\text { Saúde Coletiva }\end{array}$ \\
\hline 4 & $\begin{array}{l}\text { Intervenção interdisciplinar enquanto estratégia para o Uso Racional de } \\
\text { medicamentos em Idosos. }\end{array}$ & $\begin{array}{l}\text { Eloá Fátima Ferreira } \\
\text { Medeiros et al. }\end{array}$ & 2009 & $\begin{array}{l}\text { Revista Ciência e } \\
\text { Saúde Coletiva }\end{array}$ \\
\hline 5 & $\begin{array}{l}\text { Quedas e fatores associados em idosos institucionalizados no município } \\
\text { de Pelotas (RS, Brasil). }\end{array}$ & $\begin{array}{l}\text { Maitê Peres de } \\
\text { Carvalho et al }\end{array}$ & 2008 & $\begin{array}{l}\text { Revista Ciência } \\
\text { Saúde Coletiva }\end{array}$ \\
\hline 6 & $\begin{array}{l}\text { Capacidade funcional, condições socioeconômicas e de saúde de idosos } \\
\text { atendidos por equipes de Saúde da Família de Goiânia (GO, Brasil). }\end{array}$ & $\begin{array}{l}\text { Daniella Pires Nunes } \\
\text { et al }\end{array}$ & 2008 & $\begin{array}{l}\text { Revista Ciência } \\
\text { Saúde Coletiva }\end{array}$ \\
\hline 7 & $\begin{array}{l}\text { Violência contra a pessoa idosa: análise de aspectos da atenção de saúde } \\
\text { mental em cinco capitais brasileiras. }\end{array}$ & $\begin{array}{lr}\text { Fabianar } & \text { Castelo } \\
\text { Valadares, } & \text { Edinilsa } \\
\text { Ramos de Souza. }\end{array}$ & 2010 & $\begin{array}{l}\text { Revista Ciência e } \\
\text { Saúde Pública }\end{array}$ \\
\hline 8 & $\begin{array}{l}\text { Experiências psíquicas de mulheres frequentadores da rede pública de } \\
\text { saúde em Teresina (PI, Brasil). }\end{array}$ & $\begin{array}{l}\text { Ana Célia Cavalcante, } \\
\text { Raimunda Magalhães } \\
\text { Silva. }\end{array}$ & 2007 & $\begin{array}{l}\text { Revista Ciência e } \\
\text { Saúde Pública }\end{array}$ \\
\hline 9 & $\begin{array}{l}\text { A violência doméstica contra idosos nas áreas de abrangência do } \\
\text { Programa Saúde da Família de Niterói (RJ, Brasil). }\end{array}$ & $\begin{array}{l}\text { Paulo Cavalcante } \\
\text { Apratto Júnior }\end{array}$ & 2010 & $\begin{array}{l}\text { Revista Ciência } \\
\text { Saúde Coletiva }\end{array}$ \\
\hline 10 & Suicídio entre pessoas idosas: revisão da literatura. & $\begin{array}{l}\text { Maria Cecília de } \\
\text { Souza Minayo, Fátima } \\
\text { Gonsalves Cavalcante. }\end{array}$ & 2010 & $\begin{array}{l}\text { Revista } \\
\text { Pública }\end{array}$ \\
\hline 11 & $\begin{array}{l}\text { êmica brasileira sobre causas } \\
\text { sa. }\end{array}$ & $\begin{array}{l}\text { Maria Cecília de Souza } \\
\text { Minayo et al }\end{array}$ & 2010 & $\begin{array}{l}\text { Revista Ciência e } \\
\text { Saúde Coletiva. }\end{array}$ \\
\hline 12 & $\begin{array}{l}\text { Avaliação do projeto de promoção da saúde do Núcleo de Atenção ao } \\
\text { idoso. }\end{array}$ & Mônica de Assis et al & 2009 & $\begin{array}{l}\text { I n t e } r f \text { a c e, } \\
\text { comunicação, saúde } \\
\text { e educação. }\end{array}$ \\
\hline 13 & $\begin{array}{l}\text { Idosos vacinados e não-vacinados contra influenza: morbidade relatada } \\
\text { e aspectos sóciodemográficos, Porto Alegre - RS - Brasil. }\end{array}$ & $\begin{array}{l}\text { Maria Aparecida } \\
\text { Muller Vilarino et al }\end{array}$ & 2010 & $\begin{array}{l}\text { Revista Ciência } \\
\text { Saúde Coletiva }\end{array}$ \\
\hline 14 & $\begin{array}{l}\text { Incontinência Urinária feminina: revisão sistemática de estudos } \\
\text { qualitativos. }\end{array}$ & Celione Volkmer et al & 2012 & $\begin{array}{l}\text { Revista Ciência } \\
\text { Saúde Coletiva }\end{array}$ \\
\hline 15 & $\begin{array}{l}\text { Prevalência de idosos restritos ao domicílio em região metropolitana de } \\
\text { Belo Horizonte- Minas Gerais-Brasil. }\end{array}$ & $\begin{array}{l}\text { Priscila Guedes } \\
\text { Santana Ursine et al }\end{array}$ & 2011 & $\begin{array}{l}\text { Revista Ciência } \\
\text { Saúde Coletiva }\end{array}$ \\
\hline 16 & A realidade da saúde bucal do idoso no Sudeste brasileiro & $\begin{array}{l}\text { Ana Carolina de Assis } \\
\text { Simões; Denise Maciel } \\
\text { Carvalho }\end{array}$ & 2011 & $\begin{array}{l}\text { Revista Ciência } \\
\text { Saúde Coletiva }\end{array}$ \\
\hline 17 & $\begin{array}{l}\text { Diferenças de gênero e medicalização da sexualidade na criação do } \\
\text { diagnóstico das disfunções sexuais }\end{array}$ & Fabíola Rohden & 2009 & Estudos Feministas \\
\hline 18 & $\begin{array}{l}\text { Eventos estressantes, estratégias de enfrentamento, auto-eficácia e } \\
\text { sintomas depressivos entre idosos residentes na comunidade }\end{array}$ & $\begin{array}{l}\text { Andréa Cristina } \\
\text { Garofe Fortes-Burgos } \\
\text { et al }\end{array}$ & 2007 & $\begin{array}{l}\text { Psicologia: reflexão e } \\
\text { crítica }\end{array}$ \\
\hline 19 & $\begin{array}{l}\text { A contribuição da atenção domiciliar para a configuração de redes } \\
\text { substitutivas de saúde: desinstitucionalização e transformação de } \\
\text { práticas }\end{array}$ & $\begin{array}{l}\text { Laurar.M. } \\
\text { Feuerwerker, Emerson } \\
\text { Elias Merhy. }\end{array}$ & 2008 & $\begin{array}{l}\text { R e v i s t a } \\
\text { Panamericana } \\
\text { Salud Publica }\end{array}$ \\
\hline 20 & $\begin{array}{l}\text { Morbi-mortalidade por doenças do aparelho respiratório em idosos } \\
\text { antes e após a introdução da vacina contra Influenza }\end{array}$ & $\begin{array}{l}\text { Marilene Bibiana } \\
\text { Pereira Nieiro et al }\end{array}$ & 2008 & $\begin{array}{l}\text { São Paulo } \\
\text { Perspectiva }\end{array}$ \\
\hline 21 & $\begin{array}{l}\text { A violência contra idosos na mídia: uma reflexão sobre a produção dos } \\
\text { sentidos }\end{array}$ & $\begin{array}{l}\text { Alarcon Agra de Ó et } \\
\text { al }\end{array}$ & 2010 & Revista FAMECOS \\
\hline 22 & Diferenciais intra-urbanos de vulnerabilidade da população idosa. & $\begin{array}{l}\text { Luciana de Souza } \\
\text { Braga et al }\end{array}$ & 2010 & $\begin{array}{l}\text { Revista Ciência e } \\
\text { Saúde Coletiva }\end{array}$ \\
\hline 23 & Vulnerabilidade e envelhecimento no contexto da saúde. & $\begin{array}{l}\text { Adriana Aparecida } \\
\text { Paz et al }\end{array}$ & 2006 & $\begin{array}{l}\text { Acta Paulista } \\
\text { Enfermagem }\end{array}$ \\
\hline
\end{tabular}

